\title{
The Danish Heart Registry
}

This article was published in the following Dove Press journal:

Clinical Epidemiology

25 October 2016

Number of times this article has been viewed

\author{
Cengiz Özcan ${ }^{1,2}$ \\ Knud Juel' \\ Jens Flensted Lassen ${ }^{3}$ \\ Lene Mia von Kappelgaard' \\ Poul Erik Mortensen ${ }^{4}$ \\ Gunnar Gislason 1,2 \\ 'The National Institute of Public \\ Health, University of Southern \\ Denmark, Copenhagen K, Denmark; \\ ${ }^{2}$ Department of Cardiology, \\ Copenhagen University Hospital \\ Gentofte, Hellerup, Denmark; \\ ${ }^{3}$ Department of Cardiology, \\ The Heart Center, Copenhagen \\ University Hospital Rigshospitalet, \\ Copenhagen $\varnothing$, Denmark; \\ ${ }^{4}$ Department of Thoracic Surgery, \\ Odense University Hospital, \\ Odense C, Denmark
}

Correspondence: Cengiz Özcan

The National Institute of Public Health, University of Southern Denmark, Øster Farimagsgade 5A, I 353 København K,

Denmark

Tel +45 65507777

$\mathrm{Fax}+4539208010$

Email Ceno@si-folkesundhed.dk
Aim: The Danish Heart Registry (DHR) seeks to monitor nationwide activity and quality of invasive diagnostic and treatment strategies in patients with ischemic heart disease as well as valvular heart disease and to provide data for research.

Study population: All adult ( $\geq 15$ years) patients undergoing coronary angiography (CAG), percutaneous coronary intervention (PCI), coronary artery bypass grafting, and heart valve surgery performed across all Danish hospitals were included.

Main variables: The DHR contains a subset of the data stored in the Eastern and Western Denmark Heart Registries (EDHR and WDHR). For each type of procedure, up to 70 variables are registered in the DHR. Since 2010, the data quality protocol encompasses fulfillment of web-based validation rules of daily-submitted records and yearly approval of the data by the EDHR and WDHR.

Descriptive data: The data collection on procedure has been complete for PCI and surgery since 2000, and for CAG as of 2006. From 2000 to 2014, the number of CAG, PCI, and surgical procedures changed by $231 \%, 193 \%$, and $99 \%$, respectively. Until the end of 2014, a total of 357,476 CAG, 131,309 PCI, and 60,831 surgical procedures had been performed, corresponding to $249,445,100,609$, and 55,539 first-time patients, respectively. The DHR generally has a high level of completeness (1-missing) of each procedure (>90\%) when compared to the National Patient Registry. Variables important for assessing the quality of care have a high level of completeness for surgery since 2000, and for CAG and PCI since 2010.

Conclusion: The DHR contains valuable data on cardiac invasive procedures, which makes it an important national monitoring and quality system and at the same time serves as a platform for research projects in the cardiovascular field.

Keywords: nationwide, coronary angiography, percutaneous coronary intervention, cardiac surgery

\section{Introduction}

Around the turn of the last century, Denmark was facing a relatively high cardiovascular mortality rate and a low utilization of invasive cardiac procedures compared to other Nordic countries. ${ }^{1,2}$ Consequently, Danish health care authorities initiated a comprehensive nationwide initiative (the Heart Plan) to improve diagnosis and treatment of cardiovascular disease. In order to monitor the Heart Plan, the Danish Heart Registry (DHR) was established in 1998. The plan resulted in an increase in the use of coronary diagnostics and interventions and contributed to a continuous drop in mortality due to cardiovascular disease in Denmark. ${ }^{3}$ To ensure that patients continue to benefit from improved quality of life and survival, it is of paramount importance to surveil 
that the extent of invasive procedures outweigh the risks and document stable or improving quality of treatment throughout the country. Reporting data to the DHR was therefore made compulsory. Each operator at each hospital submits data to their local database (the East Denmark Heart Registry [EDHR] or the West Denmark Heart Registry [WDHR] ${ }^{4}$ electronic database). All procedures, but with a selected set of data variables, are subsequently extracted from the EDHR and WDHR and reported to the DHR.

In Denmark, with a total population of $5,660,000$ (January 1, 2015), a unique and personal identifier number is assigned to each individual residing in Denmark at birth or upon immigration, which enables cross-linking data from the DHR with other nationwide registries. Hence, the National Patient Registry (NPR) ${ }^{5}$ and the Danish Civil Registration System $^{6}$ are used to assess the DHR's completeness of procedures and conduct mortality analyses, respectively.

\section{Aim}

The DHR monitors the quantity and quality of invasive procedures in different patient groups across all hospitals and serves as a data source for nationwide cardiovascular research within the field of ischemic heart and heart valve disease.

\section{Study population}

The DHR has been previously described; ${ }^{7}$ however, it currently requires an expansion of other diagnostic and treatment areas to be included in the registry. In its present form, the DHR holds information on all coronary angiography (CAG), percutaneous coronary intervention (PCI), coronary artery bypass grafting (CABG), and heart valve replacement performed on patients aged $\geq 15$ years across the country. In the coming years, the DHR will also include national coverage of transcatheter aortic valve implantation (TAVI) and coronary computed tomography angiography (CCTA).

The DHR contains nationwide data on PCI and surgical activities since 2000 and CAG procedures since 2006. However, at present, only the WDHR reports TAVI to the DHR. Between 2000 and 2014, the number of CAG, PCI, and surgical procedures changed by $231 \%, 193 \%$, and $99 \%$, respectively. By the end of 2014, a total of 357,476 CAG, 131,309 PCI, and 60,831 surgical procedures had been registered in the DHR, corresponding to 249,445, 100,609, and 55,539 first-time patients, respectively. Figure 1 shows the trends of each procedure, including isolated CABG and heart valve surgery, among first-time patients.

As of 2014, 12 public and one private hospital carried out CAG across the country. Among these, PCI was performed in

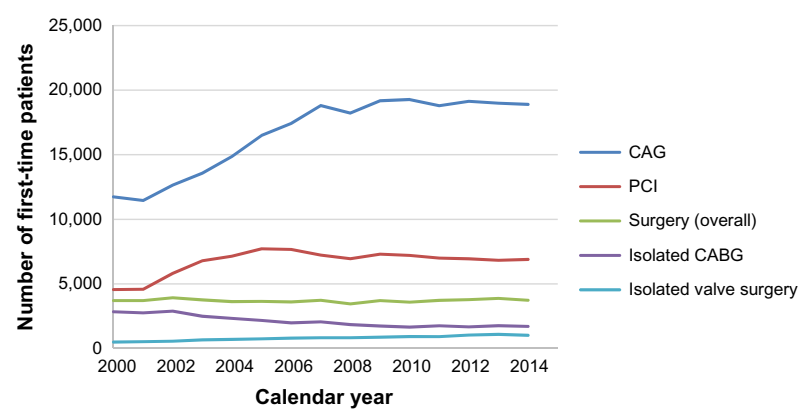

Figure I Number of first-time patients in the Danish Heart Registry who received invasive procedures from 2000 to 2014.

Abbreviations: CAG, coronary angiography; $\mathrm{PCl}$, percutaneous coronary intervention; $C A B G$, coronary artery bypass grafting.

seven centers, of which one is a private hospital with a very low number of procedures. Four centers performed primary PCI around the clock and had facilities for urgent surgery if needed. In 2014, all public PCI centers performed at least 821 PCI per center yearly. Four public and one privately funded hospital offered cardiac surgery.

In the future, the number of hospitals reporting to the DHR will increase, as hospitals performing CCTA also will contribute data to the DHR, of which the WDHR has already validated the CCTA data. ${ }^{8}$

Given the Danish reimbursement policy, hospitals are required to report procedures to the NPR as well. The completeness (1-missing) of each procedure registered in the DHR was measured as the percentage of registered procedures in the NPR, which was also registered in the DHR (Figure 2). Since the DHR released its first annual report in 2005 , covering data from 2003, a general high completeness $(>90 \%)$ of procedures has been reported, although for certain procedures a high level of completeness have been present since 2000. The NPR has national coverage on CAG from 2004, which is the earliest time from which comparison of CAG between the DHR and the NPR can be

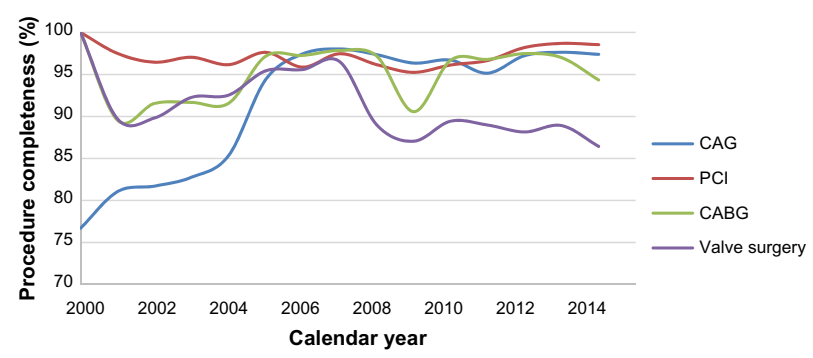

Figure 2 Trends in the percentage of procedures registered in the Danish Heart Registry as well as in the National Patient Registry in relation to the number of procedures registered in the National Patient Registry.

Notes: CABG denotes all surgical procedures that at least include CABG. Valve surgery denotes all surgical procedures that at least include valve surgery.

Abbreviations: CAG, coronary angiography; $\mathrm{PCl}$, percutaneous coronary intervention; $C A B G$, coronary artery bypass grafting. 
made. In 2008, TAVI was offered as a treatment for selected patients, but because only one regional database reported TAVI to the DHR, the consequence has been a somewhat lower estimate for valve surgery in recent years. In addition, between 2008 and 2011, the local clinical databases in East Denmark merged, resulting in missing data from one surgical hospital during 2009-2010. The data have been found and are now in the process of being loaded onto the database by the DHR's data manager. Figure 2 reflects the impact of the missing surgical data from East Denmark. In comparison, the level of completeness for CAG, PCI, $\mathrm{CABG}$, and valve surgery in the WDHR was in the range of $97 \%-98 \%$ in $2008 .^{4}$

\section{Main variables}

Each invasive procedure is described by approximately 60-70 unique variables that cover demographic characteristics, prognostic factors, and procedure-related complications. Table 1 provides an overview of the majority of the data variables registered in the DHR.

Based on these variables, an annual report is produced presenting key figures at the national and the center level. The report contains summary of activity, waiting time, and prognostic factors such as indication, age, sex distribution, diabetics, emergency procedures, use of stent, and additive European System for Cardiac Operative Risk Evaluation (EuroSCORE) II, which replaced EuroSCORE I in 2015.

The report also includes quality indicators designed to evaluate the quality of care at a center level. These indicators cover procedure-related complications in CAG, PCI, CABG, and heart valve surgery and mortality following PCI, CABG, and heart valve surgery (Table 1). For each invasive procedure up to 13 quality measures are provided and, if available, compared to performance standards, which are based on best available evidence or expert consensus for which there is no firm evidence available.

Variables describing CAG- and PCI-related complications include local vascular complications, X-ray dose, myocardial infarction, stroke, and emergency CABG surgery (for PCI only). Variables describing surgical complications comprise procedure-related myocardial infarction, stroke, length of stay in the intensive care unit, reoperations for bleeding, and deep sternal wound infection.

For each procedure, variable completeness (1-missing) was measured as the percentage of first-time patients with registered variable (Table 1). The majority of the variables reported in the annual report have a high level of completeness $(>90 \%)$. Variables with a low level of completeness
$(<90 \%)$ have a greater tendency of missing information between 2000 and 2010 (data not shown), at which point the Registry Support Centre of Clinical Quality and Health Informatics (KCKS-East) became the new data manager of the DHR. Between 2000 and 2014, variables used to assess the quality of surgical procedures had, in the majority of cases, a high level of completeness. In contrast, among the variables used to assess the quality of CAG and PCI, only X-ray dose had a high level of completeness. Since 2010, an improvement was noted across all quality variables in all procedures. The level of completeness was in the range of $91.9 \%-99.8 \%$ between 2010 and 2014 (data not shown). A previous validation study documented the low level of completeness for some variables. Between 2005 and 2006, 200 randomly selected procedures in the DHR (50 CAG, 50 PCI, $50 \mathrm{CABG}$, and 50 valve procedures) were validated against the local electronic database and the written patient records. In general, there was a good agreement, but some of the 28 variables being tested had a low level of completeness, particularly for complication variables in CAG and PCI ( $>40 \%$ of completeness). ${ }^{9}$ The occurrence of missing data was most likely because of the lack of registration rather than problems with data transfer. However, in 2008, the $\mathrm{WDHR}^{4}$ had a higher level of completeness for certain PCI variables (contrast volume and number of treated arteries: $100 \%)$ than what we reported herein $(62.3 \%$ and $77.7 \%$, respectively). Even though the WDHR covers $55 \%$ of the total Danish population, the comparison is impaired by the fact that this study was based on 2008 data only; nevertheless, this discrepancy raises concern regarding the quality of data transfer related to this period. Furthermore, since 2009, the data content has been updated. The most recent introduction of a new variable was in 2015 . Hence, a period of time will elapse before meaningful analyses can be performed for some variables.

\section{Follow-up}

Multiple mechanisms are at work to ensure the data quality. Since 2010, each regional data source submits daily record on each procedure through a secure website, the DHR web service, which automatically checks if the validation rules are met. Once a record is accepted, the sender is notified and the record being a EXtensible Markup Language file (XML) is converted to a Statistical Analysis System (SAS) file, which is subsequently loaded into the DHR database. In contrast, when rejected, the sender is notified about the reason for rejection (eg, length of intensive care unit time exceeding more than 6 months). Further, during follow-up, each hospital 
Table I The majority of the Danish Heart Registry's data elements depicting the baseline characteristics of first-time patients undergoing CAG, PCl, and/or surgery and variable completeness between 2000 and 2014

\begin{tabular}{|c|c|c|c|c|c|c|}
\hline \multirow{2}{*}{$\begin{array}{l}\text { Patient and procedure related } \\
\text { characteristics }\end{array}$} & \multicolumn{3}{|c|}{ Baseline characteristics $^{a}$} & \multicolumn{3}{|c|}{ Variable completeness } \\
\hline & CAG & $\mathbf{P C l}$ & Surgery & CAG & $\mathbf{P C l}$ & Surgery \\
\hline Number of patients & 249,445 & 100,609 & 55,539 & 249,445 & 100,609 & 55,539 \\
\hline Age, years & $63.8(12.0)$ & $64.5(11.7)$ & $65.8(11.4)$ & 100 & 100 & 100 \\
\hline Male sex, n (\%) & $23,0728(66.0)$ & $72,595(72.2)$ & $40,924(73.7)$ & 100 & 100 & 100 \\
\hline Height, cm & $172.3(9.5)$ & I73.I (9.2) & $172.6(9.2)$ & 57.1 & 54.5 & 47.9 \\
\hline Weight, kg & $81.0(17.4)$ & $81.8(16.5)$ & $80.8(16.1)$ & 57.6 & 54.9 & 48.2 \\
\hline Smoking status (current or former), n (\%) & $154,536(62.0)$ & $64,990(64.6)$ & $30,021(54.1)$ & 90.2 & 86.7 & 80.9 \\
\hline Diabetes (with or without treatment), $\mathrm{n}(\%)$ & $33,275(13.3)$ & $12,739(12.7)$ & $7,940(14.3)$ & 93.6 & 89.6 & 85.0 \\
\hline Left ventricular ejection fraction & $53.4(13.7)$ & $52.8(13.1)$ & - & 47.0 & 40.2 & - \\
\hline Cardiac centers & - & - & - & 100 & 100 & 100 \\
\hline Clinical presentation & - & - & - & 92.4 & 92.5 & - \\
\hline Acute, n (\%) & $42,787(17.2)$ & $33,012(32.8)$ & - & - & - & - \\
\hline Subacute, n (\%) & $46,761(18.7)$ & $19,758(19.6)$ & - & - & - & - \\
\hline Elective, n (\%) & $|40,82|(56.5)$ & $40,318(40.1)$ & - & - & - & - \\
\hline Unknown, n (\%) & $19,076(7.6)$ & $7,521(7.5)$ & - & - & - & - \\
\hline Indication & & & & 92.7 & 87.9 & - \\
\hline STEMI, n (\%) & $31,693(12.7)$ & 24,091 (23.9) & - & - & - & - \\
\hline NSTEMI/UAP, n (\%) & $58,787(23.6)$ & $25,433(25.3)$ & - & - & - & - \\
\hline Stable angina, $\mathrm{n}(\%)$ & $80,978(32.5)$ & $29,678(29.5)$ & - & - & - & - \\
\hline Other, n (\%) & $59,721(23.9)$ & $9,208(9.2)$ & - & - & - & - \\
\hline Unknown, n (\%) & $18,266(7.3)$ & $12,199(12.1)$ & - & - & - & - \\
\hline Referral date & - & - & - & 98.1 & 94.7 & 61.9 \\
\hline Procedure date & - & - & - & 100 & 100 & 100 \\
\hline Procedure codes (NCSP) ${ }^{\mathrm{b}}$ & - & - & - & 62.7 & 81.9 & 50.7 \\
\hline Catheter size, $\mathrm{Fr}$ & $5.9(0.4)$ & $6.0(6.0,6.0)$ & - & 61.6 & 61.3 & - \\
\hline Contrast volume, $\mathrm{mL}$ & $70.0(50.0,100.0)$ & $100.0(70.0, \mid 70.0)$ & - & 62.5 & 62.3 & - \\
\hline Fluoroscopy time, min & $2.0(1.0,4.0)$ & $6.70(3.0,12.0)$ & - & 99.2 & 99.1 & - \\
\hline X-ray dose, $\mathrm{Gy} \mathrm{cm}^{2}$ & $25.0(14.0,44.0)$ & $41.0(20.0,79.0)$ & - & 98.4 & 98.7 & - \\
\hline CAD severity & - & - & - & - & 50.5 & - \\
\hline I-vessel disease, n (\%) & 49,691 (19.9) & - & - & - & - & - \\
\hline 2-vessel disease, n (\%) & $13,706(5.5)$ & - & - & - & - & - \\
\hline 3-vessel disease, n (\%) & 15,384 (6.2) & - & - & - & - & - \\
\hline Diffuse CAD, n (\%) & $17,833(7.1)$ & - & - & - & - & - \\
\hline Normal, n (\%) & 29,414 (1I.8) & - & - & - & - & - \\
\hline Unknown, n (\%) & $123,4 \mid 7(49.5)$ & - & - & - & - & - \\
\hline Number of stents & - & $1.0(1.0,2.0)$ & - & - & 61.7 & - \\
\hline Number of treated lesions & - & $1.0(1.0,2.0)$ & - & - & 99.5 & - \\
\hline Number of treated arteries & - & - & - & - & 77.7 & - \\
\hline I vessel, n (\%) & - & $65,844(65.4)$ & - & - & - & - \\
\hline 2 vessels, $\mathrm{n}(\%)$ & - & II,I23 (II.I) & - & - & - & - \\
\hline 3 vessels, $\mathrm{n}(\%)$ & - & $\mathrm{I}, 203(1.2)$ & - & - & - & - \\
\hline Unknown, n (\%) & - & $22,439(22.3)$ & - & - & - & - \\
\hline Local vascular complications, n (\%) & $3,516(1.4)$ & $\mathrm{I}, 625(\mathrm{I} .6)$ & - & 77.5 & 80.0 & - \\
\hline Emergency CABG surgery, n (\%) & $\mathrm{I}, 555(0.6)$ & $422(0.4)$ & - & 60.7 & 60.5 & - \\
\hline Surgery & & & & - & - & 99.2 \\
\hline Isolated CABG, n (\%) & - & - & $31,663(57.0)$ & - & - & - \\
\hline Isolated valve surgery, $\mathrm{n}(\%)$ & - & - & $|2,0| \mid(2 \mid .6)$ & - & - & - \\
\hline $\mathrm{CABG}$ and valve surgery, $\mathrm{n}(\%)$ & - & - & $4,622(8.3)$ & - & - & - \\
\hline Other, n (\%) & - & - & $6,80 I(12.2)$ & - & - & - \\
\hline Unknown, n (\%) & - & - & $442(0.8)$ & - & - & - \\
\hline \multicolumn{7}{|l|}{ Valvular surgery } \\
\hline Aortic, n (\%) & - & - & $9,495(17.1)$ & - & - & 100 \\
\hline Mitral, n (\%) & - & - & $2,694(4.9)$ & - & - & 100 \\
\hline Tricuspid, n (\%) & - & - & $303(0.6)$ & - & - & 59.7 \\
\hline Pulmonary, n (\%) & - & - & $145(0.3)$ & - & - & 59.7 \\
\hline
\end{tabular}


Table I (Continued)

\begin{tabular}{|c|c|c|c|c|c|c|}
\hline & \multicolumn{3}{|c|}{ Baseline characteristics $^{a}$} & \multicolumn{3}{|c|}{ Variable completeness } \\
\hline & CAG & $\mathbf{P C l}$ & Surgery & CAG & $\mathbf{P C l}$ & Surgery \\
\hline EuroSCORE & - & - & $5.2(3.5)$ & - & - & 95.3 \\
\hline Extracorporal circulation, n (\%) & - & - & $43,168(77.7)$ & - & - & 97.0 \\
\hline ICU length of stay, hours & - & - & $22.0(20.0,24.0)$ & - & - & 86.0 \\
\hline Reoperation for bleeding, n (\%) & - & - & $3,124(5.6)$ & - & - & 94.1 \\
\hline Deep sternal wound infection, $\mathrm{n}(\%)$ & - & - & $549(1.0)$ & - & - & 92.4 \\
\hline Procedure-related myocardial & $1,619(0.6)^{c}$ & $513(0.5)^{c}$ & $1,713(3.1)^{d}$ & 79.4 & 80.1 & 91.3 \\
\hline \multicolumn{7}{|l|}{ infarction, n (\%) } \\
\hline Procedure-related stroke, n (\%) & $278(0.1)^{c}$ & $353(0.4)^{c}$ & $847(1.5)^{\mathrm{e}}$ & 62.6 & 62.3 & 91.2 \\
\hline Vital status & - & - & - & 98.3 & 98.1 & 98.3 \\
\hline
\end{tabular}

Notes: a Continuous variables are presented as means (SD) or medians (interquartile range), and categorical variables are presented as numbers (\%). ${ }^{\circ} \mathrm{NCSP}$ codes are used to classify each procedure: CAG (UXAC40, UXAC85, UXAC90, UXUC85-UXUC87, UFYA20), PCI (KFNG00, KFNG02, KFNG05, KFNGI0, KFNGI2, KFNG20, KFNG22, KFNG30, KFNG40, KFNG96, KZFX0I), CABG (KFNA-F, KFNH, KFNJ, KFNK, KFNW), valve surgery (KFG, KFK, KFJE, KFJF, KFM). 'Within I2 hours, dduring admission, eonset of symptoms within 12 hours and lasting $>24$ hours.

Abbreviations: CAG, coronary angiography; PCI, percutaneous coronary intervention; STEMI, ST-segment elevation myocardial infarction; NSTEMI, non-ST segment elevation myocardial infarction; UAP, unstable angina pectoris; NCSP, NOMESCO classification of surgical procedures; Fr, the French scale; CAD, coronary artery disease; CABG, coronary artery bypass grafting; EuroSCORE, European System for Cardiac Operative Risk Evaluation; ICU, intensive care unit; SD, standard deviation.

has access to their own data, enabling them to monitor their reports, and twice a year activity and waiting time for invasive procedures in the preceding 2-year period are presented for the National Board of Health. In addition, the yearly report undergoes critical review and approval by board members of the DHR before it is made available online. ${ }^{10}$

\section{Examples of research}

The DHR has served as a data source for several nationwide studies. Each annual report contains a list of performed and ongoing research. During 2000-2009, the number of firsttime CAG has been reported to have increased more than $50 \%$, and this was mostly attributed to patients with no prior history of ischemic heart disease. Furthermore, mean age, proportion of women, and use of prophylactic cardiovascular drugs increased as well. ${ }^{11}$ Another study assessed the national trends in time to invasive examination and treatment in non-ST segment elevation myocardial infarction (NSTEMI) and unstable angina between 2001 and 2009. This study demonstrated that utilization of CAG and PCI had increased as opposed to $\mathrm{CABG}$ and that the proportion of patients undergoing invasive examination and treatment within 3 days had increased. ${ }^{12}$ A study on first-time acute myocardial infarction without significant stenosis did not show any difference between the sexes in terms of the level of secondary preventive medicine and long-term mortality and recurrence of acute myocardial infarction. ${ }^{13}$ The Scandinavian Organization for Randomized Trials with Clinical Outcome II performed a head-to-head comparison of the clinical efficacy and safety between the first two drug-eluting stents (DES [sirolimus-eluting Cypher and the pacllitaxel- eluting Taxus stents]) in patients deemed suitable for DES and with STEMI, NSTEMI, or unstable/stable angina. No significant difference was observed after short- and longterm follow-up. ${ }^{14,15}$

\section{Administrative issues and funding}

The DHR is administered and maintained by the Danish National Institute of Public Health (NIPH) and funded by the Danish Clinical Registries (RKKP). Since 2010 and 2014, KCKS-East, which serves under RKKP, has been responsible for the data management and delivery of data for research.

The structure of the DHR organization comprises three bodies - the board, the executive steering group, and the office. The DHR board includes representatives from selected organizations (the Danish Society of Cardiology [DCS], the Danish Society of Thoracic Surgery [DTS], the Danish Society of Anaesthesiology and Intensive Care Medicine, the Danish Society of Clinical Physiology and Nuclear Medicine, the RKKP, and the NIPH) and departments performing invasive procedures. Hospitals performing only CAG are represented by two representatives, each representing the EDHR and the WDHR. The board is responsible for the overall strategy, development of goals, overseeing the organization management, approving the annual report, introducing new variables, revising the quality indicators, and updating the performance standards in collaboration with representatives from the DCS and the DTS. The board has appointed an executive steering group, which comprises the chairman, an interventionalist/surgeon, the NIPH representative, and a medical consultant. The executive steering group ensures the management of the DHR organization between the annual 
board meetings and supervises the office located at the NIPH, which is responsible for the day-to-day administrative operations, implementing the changes set forth by the executive steering committee, handling incoming and outgoing communications, organizing the annual board meeting, and preparing the annual report.

The DHR is approved by the National Board of Health and the Danish Data Protection Agency (Reference number 2007-58-0014).

\section{Conclusion}

The DHR contains valuable information on invasive cardiac procedures and serves its purpose as a national monitoring and quality control system. Cross-linking the DHR with a variety of national databases on an individual level makes it a powerful tool to secure continuous quality monitoring of cardiac interventions and surgical procedures on a nationwide level, as well as an important resource for cardiovascular research. The DHR looks forward to an expansion in the near future by including information on CCTA and TAVI, which enables monitoring of novel diagnostics and interventions within cardiovascular care. However, the DHR is also faced with other challenges, as evidence suggests previous problems with the registration and the data transfer for some variables probably until 2010, at which point a new data manager has since then ensured the data quality by automatic validation. Finally, the continued expansion of the DHR entails the risk of data break for some variables, which also needs to be addressed in the near future.

\section{Acknowledgment}

This paper was funded by the Program for Clinical Research Infrastructure (PROCRIN) established by the Lundbeck Foundation and the Novo Nordisk Foundation and administered by the Danish Regions.

\section{Disclosure}

The authors report no conflicts of interest in this work.

\section{References}

1. Nordic Medico-Statistical Committee (NOMESCO). Nordic/Baltic Health Statistics 1999. Copenhagen, Denmark: NOMESCO; 2000.

2. Technological change around the world: evidence from heart attack care. Health Aff (Millwood). 2001;20(3):25-42.

3. Jørgen Videbæk MM. 2004 Heart Statistics. Copenhagen, Denmark: Hjerteforeningen; 2004.

4. Schmidt M, Maeng M, Jakobsen CJ, et al. Existing data sources for clinical epidemiology: The Western Denmark Heart Registry. Clin Epidemiol. 2010;2:137-144.

5. Schmidt M, Schmidt SA, Sandegaard JL, Ehrenstein V, Pedersen L, Sorensen HT. The Danish National Patient Registry: a review of content, data quality, and research potential. Clin Epidemiol. 2015;7:449-490.

6. Schmidt M, Pedersen L, Sorensen HT. The Danish Civil Registration System as a tool in epidemiology. Eur J Epidemiol. 2014;29(8): 541-549.

7. Abildstrom SZ, Madsen M. The Danish Heart Register. Scand J Public Health. 2011;39(7 Suppl):46-49.

8. Nielsen LH, Norgaard BL, Tilsted HH, et al. The Western Denmark Cardiac Computed Tomography Registry: a review and validation study. Clin Epidemiol. 2015;7:53-64.

9. Abildstrom SZ, Andersen S, Hvelplund A, Rasmussen S, Krise M. The Danish Heart Register - Annual Report 2007. Copenhagen, Denmark: The National Institute of Public Health; 2008.

10. The National Institute of Public Health. The Danish Heart Registry Annual Reports. Copenhagen, Denmark: The National Institute of Public Health; 2014.

11. Jørgensen ME, Andersson C, Schjerning Olsen A-M, et al. Danish trends in pharmacotherapy, comorbidities, and demographics in patients referred for coronary angiography - what changed during a decade? Eur Heart J Cardiovasc Pharmacother. 2015;1:157-165.

12. Mårtensson S, Gyrd-Hansen D, Prescott E, Andersen PK, Zwisler A-DO, Osler M. Trends in time to invasive examination and treatment from 2001 to 2009 in patients admitted first time with non-ST elevation myocardial infarction or unstable angina in Denmark. BMJ Open. 2014;4(1):e004052.

13. Hansen KW, Hvelplund A, Abildstrom SZ, et al. No gender differences in prognosis and preventive treatment in patients with AMI without significant stenoses. Eur J Prev Cardiol. 2012;19(4):746-754.

14. Galloe AM, Thuesen L, Kelbaek H, et al. Comparison of paclitaxeland sirolimus-eluting stents in everyday clinical practice: the SORT OUT II randomized trial. JAMA. 2008;299(4):409-416.

15. Bligaard N, Thuesen L, Saunamaki K, et al. Similar five-year outcome with paclitaxel- and sirolimus-eluting coronary stents. Scand Cardiovasc J. 2014;48(3):148-155.
Clinical Epidemiology

\section{Publish your work in this journal}

Clinical Epidemiology is an international, peer-reviewed, open access, online journal focusing on disease and drug epidemiology, identification of risk factors and screening procedures to develop optimal preventative initiatives and programs. Specific topics include: diagnosis, prognosis, treatment, screening, prevention, risk factor modification,

\section{Dovepress}

systematic reviews, risk \& safety of medical interventions, epidemiology \& biostatistical methods, and evaluation of guidelines, translational medicine, health policies \& economic evaluations. The manuscript management system is completely online and includes a very quick and fair peer-review system, which is all easy to use. 